\title{
Phraseologie und Fremdsprachenlernen. Zur Problematik einer angemessenen phraseodidaktischen Umsetzung*
}

\author{
Vida Jesenšek (Maribor)
}

\begin{abstract}
In this paper the author strives for the more intensive inclusion of phraseological learning content in the teaching of foreign languages. Arguments, due to which at all levels of teaching languages should systematically provide for acquiring and respectively developing the passive and active phraseology competence, are as follow: the commonness and range of phraseological methods of expression, empirically proved acquisition of phraseological competence in the very early phase of mastering the native language and a relatively high degree of inter-language accordance of phraseological structure. In the planning of language education and the training of language teachers many consecutive changes will be necessary. Among others it will be necessary to synchronically plan teaching of the native and foreign languages, bring into force phraseology as a compulsory subject in higher education programs for the training foreign language teachers, and materially and professionally enable more intensive development of special phraseological learning material.
\end{abstract}

\section{Phraseologie und Fremdsprachenlernen}

Fakt ist, dass Phraseme ${ }^{1}$ zum lexikalischen Inventar natürlicher Sprachen gehören, dass sie Elemente der Langue sind und dass sie folglich den Sprachgebrauch (Parole) ausschlaggebend mit gestalten. Der phraseologischen Redeweise sollte man dementsprechend eine angemessene didaktische Aufmerksamkeit schenken, dies sowohl beim muttersprachlichen als auch beim fremdsprachlichen Sprachunterricht. Dem ist allerdings meist nicht so: Bereits ein paar kleinere Analysen geläufiger Lehrwerke (z. B. zum DaF) zeigen, dass die Phraseologie in quantitativer Hinsicht selten und in qualitativer Hinsicht

\footnotetext{
* Die folgenden Überlegungen resultieren größtenteils aus den Forschungsaktivitäten im Rahmen zweier Projekte, die an der Pädagogischen Fakultät der Universität Maribor (Slowenien) durchgeführt werden. Das slowenische nationale Projekt mit dem Titel Besedoslovne spremembe slovenskega jezika skozičas in prostor (J6-6284; 2004-2007) untersucht die Lexik der slowenischen Sprache unter der historischen und kontrastiven Perspektive und will u. a. theoretisch-methodologische Grundlagen schaffen für die Kontrastierung slowenischer Phraseologie mit der Kontaktsprache Deutsch, während das internationale EU-Sokrates-Projekt EPHRAS. Ein mehrsprachiges phraseologisches Lernmaterial auf CD-ROM (117024-CP-1-2004-1-SI-LINGUA-L2; 20042006) zum Hauptziel hat, ein mehrsprachiges phraseologisches Lernmaterial zu entwickeln, basierend auf einer deutsch-slowenisch-slowakisch-ungarischen Phraseologie-Datenbank. Mehr zum letztgenannten Projekt unter http;//www.ephras.org.

${ }^{1}$ Unter "Phrasem" werden in diesem Beitrag Mehrwortverbindungen verstanden, die neben der Polylexikalität und Festigkeit auch die Eigenschaft der Idiomatizität aufweisen (Phraseme im engeren Sinne, cf. Burger 2003).
} 
wenig systematisch behandelt wird (Köster 1998; Jesenšek 2000). Zudem vertritt die fremdsprachliche Didaktik zur Ermittlung von Phraseologie (noch immer) verschiedene, mitunter sogar konträre Meinungen (Hallsteinsdóttir 2001). Im Folgenden wird die Auffassung vertreten, dass der Phraseologie im gesamten Sprachunterricht von Anfang an ein fester Platz einzuräumen ist (cf. ähnliche Positionen bei Kühn 1992; Köster 1998; Jesenšek 2000; Hallsteinsdóttir 2001; Kržišnik 2001; Petermann 2001). So ist eine systematische Förderung der (passiven und aktiven) phraseologischen Kompetenz beim Sprachenlernen notwendig, um einigen deklarierten Zielen des Fremdsprachenlernens gerecht zu werden, u. a. dem handlungsorientierten Ansatz im Fremdsprachenunterricht (Glabionat et al. 2005). Klar ist, dass sich aus einer solchen Position zum Status der Phraseologie im Sprachenlernen mehrere Schlussfolgerungen ableiten lassen. Sie betreffen die gesamte (synchronisierte) Planung des Sprachunterrichts, ebenso die Fremdsprachenlehrerausbildung und die Entwicklung von Lehr- und Lernmaterialien (ausführlicher dazu Jesenšek im Druck a).

\section{Argumente für eine intensivere Förderung der phraseologischen Kompetenz}

Argumente, die für eine intensive und systematische Einbeziehung der Phraseologie in das gesamte Sprachenlernen sprechen, gibt es mehrere. Die wichtigsten werden hier in Kürze dargelegt.

Argument 1: Eine phraseologische Redeweise ist ein Normalfall der geschriebenen und gesprochenen Sprache, Phraseme sind aus dem täglichen Sprachgebrauch in verschiedensten Kontexten nicht wegzudenken. Dies gilt sprachenübergreifend und ist primär darin begründet, dass die Phraseologie oft auf Metaphorisierungsprozessen beruht und Metaphern eine übliche Art der Versprachlichung von menschlicher Umwelt, Eigenschaften und Verhaltensweisen darstellen. Psychologisch-kognitiv orientierte Richtungen in der Phraseologieforschung sprechen davon, dass Menschen die Welt auf der Basis ihrer naiven nicht-wissenschaftlichen Vorstellungen und Erfahrungen wahrnehmen und sie dann nach Ähnlichkeitsprinzipien ordnen, systematisieren und zu verstehen versuchen. Man nennt dies Konzeptualisierung. Wahrnehmungskonzepte spiegeln sich in der Sprache wider und sind somit an der Bedeutung sprachlicher Einheiten interpretierbar (cf. u. a. Lakoff 1987; Dobrovol'skij 1995; Jesenšek 2004). Eine phraseologische Redeweise ist also u. a. in der Art begründet, wie Menschen die Umwelt und sich selbst auffassen, wie sie die Wahrnehmungen zu verstehen versuchen und wie sie sie verbalisieren. Damit wird die Phraseologie zu einem konstituierenden Bestandteil der sprachlichen Kommunikation. Sie im Sprachenlernen zu vernachlässigen oder sogar zu ignorieren, wäre wider die Natur der lebendigen Sprache.

Argument 2: Phraseme sind im Gedächtnis eines Menschen verankert und zwar schon seit sehr frühen Phasen des muttersprachlichen Spracherwerbs. Nach Ergebnissen der Spracherwerbsforschung folgt die Entwicklung der muttersprachlichen phraseologischen Kompetenz der allgemeinen kognitiven Entwicklung, sodass Kinder schon sehr früh, etwa im Kindergartenalter, Phraseologie verstehen und gebrauchen können (cf. u. a. Häcki Buhofer 1997, Ďurčo 2004, Dürring 2004). Phraseme werden somit nicht erst in einer relativ späten Entwicklungsstufe des Spracherwerbs verstanden und gelernt, wie es die so genannte 
Entwicklungsstufen-These behauptet (cf. Häcki Buhofer 1997) und wie es auch in der Fremdsprachendidaktik öfters vertreten wird. Es konnte nämlich nachgewiesen werden, dass man sie in der Muttersprache in jeder Entwicklungsstufe erwirbt (cf. die Lexikon-These bei Häcki Buhofer 1997). Darüber hinaus zeigen Resultate empirischer Untersuchungen, dass der Phraseologieerwerb überwiegend direkt verläuft, und weniger über den Umweg eines bewussten Nachvollziehens metaphorischer Prozesse, worin die phraseologische Redeweise begründet ist. Die Aneignung von muttersprachlicher Phraseologie verläuft parallel mit der Aneignung von Einzelwörtern und ist nicht an ein bestimmtes Alter bzw. an eine höhere kognitive Entwicklungsstufe gebunden. Auch aus psychologisch-kognitiven Gründen wäre also angemessen, die Phraseologie didaktisch entsprechend aufzufassen und als Unterrichtsgegenstand zu etablieren (cf. u. a. Häcki Buhofer 1997; Hessky 1997; Ďurčo 2004; Dürring 2004; Jesenšek im Druck a, Šajánková 2005).

Argument 3: Stimmen wir den Postulaten der kognitiven Semantik nach Lakoff und Johnson (1980) zu, wonach wir die Welt primär metaphorisch wahrnehmen und konzeptualisieren, so ist die bildlich-metaphorische Motiviertheit vieler Phraseme darin begründet. Die idiomatische Ausdrucksweise gehört somit zu Eigenschaften natürlicher Sprachen, was u. a. zur Folge hat, dass relativ viele Phraseme zwischensprachlich hochgradig konvergent sind.2 Die formale (und weitgehend auch semantische) zwischensprachliche Konvergenz gilt in der Regel für Phraseme, die als Komponente eine Körperteilbenennung haben, bei der die Metaphorisierung auf biologischen Gegebenheiten und menschlich universellen Welterfahrungen basiert und somit nicht als einzelsprachspezifisch gedeutet werden kann. Weitere konvergente Gruppen bilden Phraseme, (1) die in einer gemeinsamen (z. B. europäischen) Kultur wurzeln, (2) die von der klassischen europäischen Literatur ausgehen und (3) die durch Massenmedien verbreitet werden. Für sie alle zeigt sich, dass sie leicht die Grenzen einzelner Sprachen überschreiten. Somit ist es kaum angemessen, die Phraseologie zur Eigentümlichkeit und Spezialität jeder Einzelsprache zu erklären. Darüber hinaus ist es längst anerkannt, dass die Muttersprache im Prinzip immer ein wichtiger Faktor beim Fremdsprachenlernen ist, auf den man als Lerner zurückgreift, bewusst oder unbewusst und nicht nur in den Anfangsphasen des Fremdsprachenlernens (Ďurčo 2004). So werden die muttersprachlichen phraseologischen Kenntnisse auf die fremdsprachliche Phraseologie übertragen und der muttersprachlichen Phraseologie kommt eine entscheidende Rolle beim Verstehen fremdsprachlicher Phraseme zu (cf. u. a. Hallsteinsdóttir 2001). Beim fremdsprachlichen Lernen entsteht dadurch im Sprachbewusstsein der Lerner ein System von lexikalischen Korrelationen zwischen Sprachen, die parallel als zwei lexikalische Systeme nebeneinander vorhanden sind (Ďurčo 2004). Die muttersprachlichen phraseologischen Kenntnisse sollten deshalb intensiver ausgenutzt werden. Einerseits aus phraseologischtheoretischen Gründen, wonach Phraseologien verschiedener Sprachen viel mehr

\footnotetext{
2 Der Begriff der Konvergenz wird in diesem Beitrag als "zwischensprachliche Korrelativität der konkreten Phraseologismen verstanden, d. h. Ähnlichkeit ihrer Sinn- oder formalen Sinnorganisation" (A. D. Rajchštejn, zitiert nach Eismann 1989, 86). Zwischen Deutsch und Slowenisch beträgt sie bei einigen Gruppen von Phrasemen bis zu 50 \% (Jesenšek 2003).
} 
Gemeinsames aufweisen als traditionell behauptet worden ist (cf. u. a. Hessky 1987; Korhonen 1997; Jesenšek 2003a). Andererseits aus psychologisch-kognitiven Gründen, wonach Phraseme ein integrierter Bestandteil des Wortschatzes seit sehr frühen Phasen des muttersprachlichen Spracherwerbs sind, weswegen sie als "Normalfall" der Rede gelten müssen. Nicht zuletzt hat die Kontrastierung der Fremdsprache mit der jeweiligen Muttersprache eine intensivere Beschäftigung mit der letzteren zur Folge, was an sich als positiv einzuschätzen ist.

\section{Entwicklung von mehrsprachigen phraseologischen Lern- und Lehrmaterialien}

Wird der Faktor Muttersprache als eine wesentliche Größe verstanden, die das Fremdsprachenlernen linguistisch und psychologisch in allen Lernstufen mitbestimmt, so ist dem auch didaktisch nachzugehen, und zwar so, dass sprachvergleichende spezielle phraseologische Lernmaterialien entwickelt und erstellt werden. Solche sind erwünscht, die ausgewählte Bereiche der jeweiligen muttersprachlichen Phraseologie den entsprechenden fremdsprachlichen Phraseologien gegenüberstellen und dadurch auf Übereinstimmungen und Unterschiede deutlich aufmerksam machen. So hat der Fremdsprachenlerner immer die Möglichkeit, auf die muttersprachliche Phraseologie zurückzugreifen. Weiter sollen solche Lernmaterialien entsprechend didaktisch aufbereitet werden, um die Entwicklung einer aktiven phraseologischen Kompetenz unterstützen zu können.

Einen Versuch in diese Richtung stellt das Forschungsprojekt EPHRAS dar (cf. http://www.ephras.org). Das Projekt hat sich zum Ziel gesetzt, ein mehrsprachiges deutschslowenisch-slowakisch-ungarisches phraseologisches Lernmaterial auf CD-ROM zu erarbeiten. Dadurch soll der derzeitige Mangel an phraseologischen Lernmaterialien der beteiligten Sprachen im Fremdsprachenunterricht behoben und zugleich ein Desiderat im Bereich der mehrsprachigen Lernmaterialien erfüllt werden. Die geplanten Lernmaterialien zielen primär auf den fremdsprachigen Lerner, der eine (oder mehrere) der vertretenen Sprachen als Fremdsprache lernt und zugleich Muttersprachler einer der vertretenen Sprachen ist. Er sollte sie auf verschiedenen Niveaustufen (B1 bis C1) sowohl zu Zwecken der Entwicklung und/oder Erweiterung von Fremdsprachenkompetenz wie auch zu Konsultationszwecken in rezeptiver und produktiver Hinsicht benutzen. Ein solches Produkt hilft, lexikalisch-phraseologische Wissenslücken zu schließen und es ermöglicht einen systematischen Erwerb und eine Erweiterung der Fremdsprachenkenntnisse im Bereich der Phraseologie. Außerdem kann es beim Textverstehen, bei der Textproduktion und beim Übersetzen als Hilfsmittel eingesetzt werden.

Im Folgenden werden zwei Dimensionen der EPHRAS-Materialien näher dargestellt: (1) Kriterien der Auswahl fremdsprachendidaktisch relevanter Phraseologie und (2) linguistische Aufbereitung der selektierten Phraseme.

\subsection{Zur Auswahl der didaktisch relevanten Phraseologie}

Eine systematische und methodologisch ausgerichtete Diskussion darüber, wie ein repräsentativer Ausschnitt aus der Phraseologie einer Sprache für (fremd)sprachendidaktische 
Zwecke zusammenzustellen ist, fehlt zur Zeit weitgehend (Lüger 1997; Jesenšek im Druck b). Dies mag mehrere Gründe haben. Zum einen liegt es daran, dass die (fremd)sprachendidaktische Perspektive keine generelle und allgemein gültige Antwort auf die Frage der Phrasem-Selektion zulässt, da "der phraseologische Lehr- und Lernstoff bei verschiedenen Lernergruppen erhebliche Unterschiede aufweisen kann bzw. muss, vor allem in Abhängigkeit von den Zielen des Unterrichts (und der Muttersprache des Lerners)" (Hessky 1997: 139). Zum anderen ist eine methodologisch begründete Festlegung der didaktisch relevanten Phraseologie prinzipiell erschwert durch die Tatsache, dass das phraseologische Inventar einer jeden Sprache dynamisch ist. Somit stellt es notwendigerweise eine offene Menge von phraseologischen Einheiten dar und ermöglicht grundsätzlich keine endgültige Auswahl. Ansatzweise sind methodologische Grundlagen zur Selektion der didaktisch relevanten Phraseologie (Sprichwörter sind darin nicht einbegriffen) trotzdem bei Koller (1977); Kühn (1992 und 1994), Dobrovol'skij (1997), Hessky (1997) und Šajánková (2005) zu finden. Allen gemeinsam ist, dass sie, beim Versuch, eine nachvollziehbare und anwendbare Selektionsmethodologie festzulegen, einen Komplex von Kriterien berücksichtigen. Dabei spielen folgende Aspekte eine unterschiedlich gewichtige Rolle: Phrasem-Definition, Bekanntheit, Frequenz, Gebräuchlichkeit, kommunikative Potenz und zwischensprachlicher Vergleich (meist mit der Muttersprache des Lerners).

Auch im Rahmen des EPHRAS-Projektes wurden Phraseme für das geplante Lernmaterial nach mehreren Selektionskriterien und anhand einer methodologisch komplexer Vorgehensweise ausgewählt, alles allerdings vor dem Hintergrund des anvisierten Lerners. Vollständigkeit konnte somit nicht angestrebt werden, obgleich dies in rezeptiver Hinsicht vielleicht von Vorteil wäre. Das systematische Auswahlverfahren richtete sich nach (1) Aspekten der Funktionalität, (2) der thematischen Relevanz für das Sprachenlernen und (3) der Aktualität.

In Bezug auf den Aspekt der Funktionalität sollte die Auswahl so ausfallen, dass der Lerner das Material sowohl zu Zwecken einer systematischen Aneignung der phraseologischen Kenntnisse als auch in Konsultationssituationen bei der Textlektüre und/oder Textproduktion brauchbar findet. Damit aufs Engste verbunden sind thematische Dimensionen der Auswahl, wonach die ausgewählten phraseologischen Materialien curricular geregelte Wortschatzbereiche für den fremdsprachlichen Unterricht im Wesentlichen decken sollten. Für den DaF-Bereich sind solche Wortschatzbereiche u. a. festgelegt und herausgearbeitet im Referenzwerk Profile Deutsch (Glabionat et al. 2005). Die darin präsentierten niveaudifferenzierten Lernzielbestimmungen und Kann-Beschreibungen beinhalten u.a. sprachliche Mittel, mit Hilfe deren man zum sprachlichen Handeln befähigt wird. So kann auch die Phrasemselektion für didaktische Zwecke auf einem handlungsorientierten Ansatz basieren, denn zur Durchführung fremdsprachendidaktisch relevanter Gruppen von Sprachhandlungen tragen unter anderen sprachlichen Mitteln auch Phraseme bei (cf. ausführlicher dazu Jesenšek im Druck b). Schließlich sind Aspekte der Aktualität wichtig, wonach die Phraseme so zu selektieren sind, dass die Auswahl für die Mehrheit der Sprecher einer Sprache im Sinne einer intersubjektiven Geläufigkeit repräsentativ ausfällt. Es kommen 
also Phraseme in Frage, für die man annehmen kann, dass sie dem überwiegenden Teil der Sprecher einer Sprache durch den häufigen Gebrauch als vorgefertigte polylexikalische Wortschatzeinheiten bekannt sind. Das Kriterium der Bekanntheit korrespondiert dabei mit dem Kriterium der Frequenz, denn prinzipiell sollte gelten: Je höher die Frequenz eines Phrasems, desto höher auch sein Bekanntheits- und Geläufigkeitsgrad. In den beiden Größen sehe ich eine brauchbare Hilfe bei der Ermittlung der fremdsprachendidaktisch relevanten Phraseologie. Im vorliegenden Text subsumiere ich die beiden Kriterien unter der Bezeichnung Aktualität. Zumal man im Fremdsprachenunterricht deklarativ in erster Linie aktuelle Fremdsprachenkentnisse anstrebt, scheinen Aspekte der Aktualität bei der Selektion von Phrasemen für wissens- und textbezogene Funktionen eines phraseologischen Lernmaterials sogar entscheidend zu sein.

Für das Deutsche finden sich empirisch überprüfte Inventarisierungen von gegenwärtig geläufigen Phrasemen nur ansatzweise, z. B. bei Dobrovol'skij (1997: 263-288) und Šajánková (2005). Beide Autoren kommen zu einer Liste von je ca. 1000 deutschen Phrasemen anhand der Methode der aktiven und passiven Informantenbefragung. Bei der aktiven Befragung wurden Informanten (vorrangig deutsche Muttersprachler) gebeten, Phraseme aufzuzählen, die sie selbst oft gebrauchen. Bei der passiven wurden ihnen vorgefertigte Phrasemlisten vorgelegt, üblicherweise das Ergebnis von Analysen von Wörterbüchern und Texten, und die Informanten mussten den Geläufigkeitsgrad einzelner Phraseme einschätzen. Auf der Liste von Šajánková basieren auch die EPHRAS-Materialien, wobei zusätzliche Selektionsschritte gemacht worden sind (Beurteilung der Gebräuchlichkeit durch deutschsprachige Germanisten, Erhebung des Gebrauchs in Österreich und in der Schweiz, danach erfolgte Korrektur durch Streichen von Phrasemen mit sehr niedrigen Bekanntheitswerten und Einfügen österreichischer und schweizerdeutscher Varianten).

Es ist allerdings bei solchen Verfahren deutlich geworden, dass endgültige, von allen Informanten akzeptierte Listen geläufiger Phraseme nicht möglich sind, vor allem deshalb nicht, weil noch immer zu wenig bekannt ist, wie Phraseme im mentalen Lexikon einzelner Sprecher funktionieren, dies sowohl in rezeptiver als auch in produktiver Hinsicht. Die Befragungsmethode verspricht allerdings in Kombination mit anderen Verfahren weiterführende Ergebnisse. In Bezug auf die Größen geläufig, weniger oder nicht geläufig sollte die phraseographische Diskussion künftig stets in Verbindung mit der Diskussion über die Brauchbarkeit und Leistungsfähigkeit von textuellen Sprachkorpora verlaufen, da diese als solide Grundlage zur Ermittlung von aktuellen Phrasemen der gegenwärtigen Sprachverwendung dienen können.

\subsection{Zur linguistischen Aufbereitung der Phraseologie für fremdsprachendidaktische Zwecke}

Dass eine einfache lexikographische Präsentation von Phrasemen, die sich etwa auf die Bedeutungserklärung und Äquivalenzangaben beschränkt, den Zwecken des Sprachenlernens nicht optimal dient, wird in der gängigen phraseologischen Literatur betont; Phraseme als lexikographische Einheiten sind in äußerst komplexen Beschreibungsdimensionen zu erfassen 
(cf. u. a. Hessky 1992; Worbs 1994; Czochralski/Ludwig 1996). Dementsprechend wurde für die Erstellung der phraseologischen Lernmaterialien ein einheitlicher Beschreibungsmodus entwickelt. Dieser basiert auf dem Mehrebenen-Matrixmodell für ein- und zweisprachige phraseologische Wörterbücher von Ďurčo (1992), welches unter Berücksichtigung einiger weiterer phraseographischer Beschreibungsansätze (cf. Dobrovol'skij 1992; Cheon 1998; Petermann 1983 und 2001; Wotjak 1989) adaptiert wurde. Das modular angelegte Mehrebenenmodell umfasst formal-strukturelle, grammatische, semantische, stilistische und kommunikativ-pragmatische Informationen zu einzelnen Phrasemen.

$\mathrm{Zu}$ den formal-strukturellen und grammatischen Angaben ist Folgendes zu sagen: Im Prinzip wird die in den traditionellen Wörterbüchern übliche Nennform von Phrasemen beibehalten: Inf. Präs. für verbale Phraseme mit persönlichem Subjekt ohne morphosyntaktische Restriktionen (in gute Hände kommen), Nom. Sg. für substantivische Phraseme ohne morphosyntaktische Restriktionen (blinder Passagier) und Nom. Sg. Mask. für adjektivische Phraseme (dünn gesät). Liegen morphosyntaktische Restriktionen vor, wird die einzig oder am häufigsten realisierte Form des Phrasems zur Nennform (jmdm. vergeht das Lachen). Da jedoch die Frage nach obligatorischen und nicht-variablen bzw. nach fakultativen und/oder variablen Komponenten eines Phrasems für den Fremdsprachenunterricht als äußerst bedeutend angesehen wird, gehören modellgerecht zu jedem Phrasemeintrag auch explizite Angaben zur eventuellen internen Varianz, zur textuell üblichen Realisierung und zur syntaktisch festgelegten externen Valenz. So wird das Phrasem keinen Finger rühren in formal-struktureller Hinsicht folgendermaßen präsentiert: keinen Finger rühren (= lexikographische Nennform), jemand ('eine Person, Gruppe von Personen') / etwas ('eine Institution') rührt keinen Finger (für jemanden/etwas) (= Angabe der externen Valenz); keinen Finger krümmen, keinen Finger krumm machen (= Angabe der internen Varianz); (etwas tun), ohne (auch nur) einen Finger zu rühren, kaum einen Finger rühren (= Angabe des typischen lexikalischen Umfeldes im Text).

Semantische, stilistische und kommunikativ-pragmatische Angaben sind in engster Beziehung zueinander zu betrachten. Angaben semantischer Art enthalten eine Bedeutungsbeschreibung des jeweiligen Phrasems (keinen Finger rühren: 'jemandem nicht helfen, untätig bleiben, sich für jemanden nicht einsetzen'), samt Angabe von phraseologischen und/oder nichtphraseologischen Quasi-Synonymen (keinen Finger rühren: keine Hand rühren, untätig bleiben, nichts tun). Stilistische Angaben markieren Phraseme hinsichtlich ihrer situativen und textuellen Verwendbarkeit (z. B. formell, informell, primär gesprochen, primär geschrieben) und/oder sie machen auf emotiv-evaluative Dimensionen der PhrasemBedeutung aufmerksam (z. B. abwertend). Pragmatisch ausgerichtete Angaben beinhalten schließlich Informationen zu den kontextuellen Umständen der Phrasemverwendung (z. B. zu den präferierten kommunikativen Situationen und Domänen, in denen ein Phrasem typischerweise verwendet wird, zur eventuellen Textsortengebundenheit, zur Sprechereinstellung und zur durch das Phrasem ausgedrückten Illokution (keinen Finger rühren: Wird häufig als Vorwurf oder Kritik an jmdn. gerichtet, der dem Sprecher bei der Arbeit oder bei Auseinandersetzungen nicht geholfen oder ihn nicht unterstützt hat). 
Nicht zuletzt ist zu betonen, dass die hier nur kurz skizzierte linguistische Aufbereitung der ausgewählten Phraseme auch Belegangaben enthält. Die kontextuellen Belege dienen sowohl der Illustration als auch der Erläuterung der linguistischen Beschreibung. Sie werden prinzipiell den verfügbaren Textkorpora der beteiligten Sprachen entnommen und sollten somit den aktuellen Sprachgebrauch repräsentieren. Für eine systematischere und effektive Entwicklung der phraseologischen Kompetenz ist notwendig, dass die Phraseologie prinzipiell in genügend umfassenden Kontexten, d. h. in textuellen und somit funktionalen Zusammenhängen, behandelt wird. Dies deshalb, weil Phraseme in der Rede, im Sprachgebrauch, ja prinzipiell im Text eingebettet und nicht isoliert vorkommen und auch deshalb, weil der entsprechende Kontext beim Verstehen, d. h. bei der Konstruktion der Bedeutung Hilfe leistet (cf. Hallsteinsdóttir 2001). Eine isolierte Behandlung von Phrasemen würde dagegen eine völlig künstliche, unnatürliche Situation herbeiführen.

\section{Zur Bedeutung zwischensprachlicher Äquivalenz im Sprachenunterricht}

Was bei der Konzeption eines solchen phraseologischen Lernmaterials eine sehr wichtige Rolle spielt, ist der Begriff der funktionalen Äquivalenz (cf. Dobrovol'skij 1999). Funktionale Äquivalenzbeziehungen sind für einen nicht-muttersprachlichen Benutzer von zentraler Bedeutung, zumal festgestelt worden ist, dass die Muttersprache prinzipiell ein wichtiger Faktor beim Fremdsprachenlernen ist, und dass die muttersprachlichen phraseologischen Kenntnisse auf die fremdsprachliche Phraseologie übertragen werden.

Theoretisch gesehen ist ein funktionales L2-Äquivalent eines L1-Phrasems ein solches, das in denselben kommunikativen Situationen gebraucht werden kann, in denen auch das L1Phrasem gebraucht wird (cf. Dobrovol'skij 1999: 114). Daraus folgt, dass funktionale Äquivalente nicht unbedingt phraseologische Systemäquivalente sein müssen, denn "ein gutes L2-Äquivalent des L1-Idioms braucht kein Idiom zu sein" (ebenda), möglicherweise ist es auch ein Einwortlexem oder eine Bedeutungsparaphrase. Dies ist besonders dann wichtig, wenn sich in der L2-Phraseologie keine Systemäquivalente des L1-Phrasems finden. Folglich werden in den geplanten phraseologischen Lernmaterialien keine einfachen 1:1-Äquivalenzen zwischen den Sprachen angestrebt, sondern es wird vielmehr versucht, 1:mehrÄquivalentbeziehungen aufzudecken und offen zu legen. Dies korrespondiert deutlich mit den Thesen, dass Phraseme übliche Elemente des Wortschatzes sind, die im Sprachunterricht parallel mit der Einwortlexik zu behandeln sind, und dass die muttersprachliche phraseologische Kompetenz positive Auswirkungen auf das Fremdsprachenlernen haben kann.

\section{Literaturangaben}

Burger, Harald (2003). Phraseologie. Eine Einführung am Beispiel des Deutschen. Berlin. Cheon, Mi-Ae (1998): Zur Konzeption eines phraseologischen Wörterbuchs für den Fremdsprachler. Am Beispiel Deutsch-Koreanisch. Tübingen. 
Czochralski, Jan A./Ludwig, Klaus-Dieter (1996): "Zur Arbeit an einem phraseologischen Wörterbuch Deutsch-Polnisch. Ein Werkstattbericht". In: Wiegand, Herbert Ernst (ed.): Studien zur zweisprachigen Lexikographie mit Deutsch III. Hildesheim/New York: 171-187.

Dobrovol'skij, Dmitrij (1992): "Zur deutschen Phraseographie". Beiträge zur Lexikologie und Lexikographie des Deutschen 33: 161-172.

Dobrovol'skij, Dmitrij (1995): Kognitive Aspekte der Idiom-Semantik, Studien zum Thesaurus deutscher Idiome. Tübingen.

Dobrovol'skij, Dimitrij (1997): Idiome im mentalen Lexikon. Ziele und Methoden der kognitiv basierten Phraseologieforschung. Trier.

Dobrovol'skij, Dmitrij (1999): "Kontrastive Phraseologie in Theorie und Wörterbuch". In: Baur, Rupprecht S./Chlosta, Christoph/Piirainen, Elisabeth (eds.): Wörter in Bildern Bilder in Wörtern. Beiträge zur Phraseologie und Sprichwortforschung aus dem Westfälischen Arbeitskreis. Baltmannsweiler: 107-122.

Ďurčo, Peter (1992): "Ein Matrixmodell für ein- und zweisprachige phraseologische Wörterbücher". In: Universität Innsbruck (ed.): Neue Ansätze in der Kontrastiven Linguistik. Proceedings of the Conference Held at the Leopold-Franzens-University of Innsbruck, Austria, 10-12 May 1991. Innsbruck: 261-269.

Ďurčo, Peter (2004): "Interferenzbereiche der Fremdsprachenphraseologie". In: Palm-Meister, Christine (ed.): Europhras 2000. Internationale Tagung zur Phraseologie vom 15.-18. Juni 2000 in Aske/Schweden. Tübingen: 79-88.

Dürring, Alexia (2004): "Das Phraseologieverständnis von Zweitklasslern - eine empirische Untersuchung". In: Palm-Meister, Christine (ed.): Europhras 2000. Internationale Tagung zur Phraseologie vom 15.-18. Juni 2000 in Aske/Schweden. Tübingen: 69-78.

Eismann, Wolfgang (1989): "Zum Problem der Äquivalenz von Phraseologismen". In: Gréciano, Gertrud (ed.): Europhras 88. Phraséologie Contrastive. Actes du Colloque International, Klingenthal-Strasbourg 12-16 mai 1988. Strasbourg: 83-93.

Glabionat, Manuela et al. (2005): Profile Deutsch. Version 2.0. Berlin etc.

Häcki Buhofer, Annelies (1997): "Phraseologismen im Spracherwerb". In: Wimmer, Rainer/ Berens, Franz-Josef (eds.): Wortbildung und Phraseologie. Tübingen: 209-232.

Hallsteinsdóttir, Erla (2001): Das Verstehen idiomatischer Phraseologismen in der Fremdsprache Deutsch. Hamburg. http://www.verlagdrkovac.de/0435_volltext.htm.

Hessky, Regina (1992): "Phraseolexeme als harte Nuß für die zweisprachige Lexikographie". In: Földes, Csaba (ed.): Deutsche Phraseologie in Sprachsystem und Sprachverwendung. Wien: 107-124.

Hessky, Regina (1997): "Einige Fragen der Vermittlung von Phraseologie im Unterricht Deutsch als Fremdsprache". In: Wimmer, Rainer/Berens, Franz-Josef (eds.): Wortbildung und Phraseologie. Tübingen: 245-261.

Hessky, Regina (1999): "Phraseologie: Vermittlungsinstanz zwischen Sprachsystem und Sprachverwendung". In: Fernandez Bravo, Nicole/Behr, Irmtraud/Rozier, Claire (eds.): Phraseme und typisierte Rede: Tübingen: 233-241. 
Jesenšek, Vida (2000): "Frazeologija in horoskop pri pouku nemškega jezika". Vestnik 34/1-2: 35-42.

Jesenšek, Vida (2003a): "Aus Nachbars Garten oder was anders? Gründe und Ursachen der zwischensprachlichen phraseologischen Konvergenz am Beispiel des Deutschen und Slowenischen". In: Teržan-Kopecky, Karmen/Petrič, Teodor (eds.): Germanistika v stičnem prostoru Evrope II, mednarodni simpozij, Maribor/Ljubljana, 18.-20. aprila 2002. Maribor: 449-455.

Jesenšek, Vida (2003b): "Dejavniki medjezikovnega frazeološkega ujemanja". Riječ 9, 1: $25-33$.

Jesenšek, Vida (2004): "Deutsch-slowenische Phraseologie aus kognitiver Perspektive". In: Bračič, Stojan et al. (eds.): Linguistische Studien im Europäischen Jahr der Sprachen. Akten des 36. Linguistischen Kolloqiums in Ljubljana 2001. Frankfurt am Main etc.: 283-290.

Jesenšek, Vida (im Druck a): "Phraseologie in der Fremdsprache Deutsch". In: Krumm, HansJürgen/Portmann-Tselikas, Paul R. (eds.): Begegnungssprache Deutsch, XIII. Internationale Deutschlehrertagung Graz. Wien.

Jesenšek, Vida (im Druck b): "Aspekte der Phrasemselektion für didaktische Zwecke. Methodische Überlegungen". Germanistische Linguistik, Zweisprachige Lexikographie und Deutsch als Fremdsprache. 3. Kolloquium zur Lexikographie und Wörterbuchforschung, Varna, 22.-24. Oktober 2004.

Koller, Werner (1977): Redensarten. Linguistische Aspekte, Vorkommensanalysen, Sprachspiel. Tübingen.

Köster, Lutz (1998): "Phraseolexeme in Horoskopen. Funktionale Analyse und didaktische Potenz dieser Textsorte für die Vermittlung von Deutsch als Fremdsprache". In: Wirrer, Jan (ed.): Phraseologismen in Text und Kontext. Phrasemata I. Bielefeld: 97-120.

Kržišnik, Erika (2001): "Vsebina (slovenskega) frazeološkega slovarja za tujce - določitev in preizkus meril". Skripta 5. Zbornik za učitelje slovenščine kot drugegaltujega jezika. Ljubljana: 7-21.

Kühn, Peter (1992): "Phraseodidaktik. Entwicklungen, Probleme und Überlegungen für den Muttersprachenunterricht und den Unterricht DaF". Fremdsprachen lehren und lernen 21: 169-189.

Kühn, Peter (1994): "Pragmatische Phraseologie: Konsequenzen für Phraseographie und Phraseodidaktik". In: Sandig, Barbara (ed.): Europhras 92. Tendenzen der Phraseologieforschung. Bochum: 411-428.

Lakoff, George (1987): Women, fire and dangerous things. What categories reveal about the mind. Chicago/London.

Lakoff, George/Johnson, Mark (1980): Metaphors we live by. Chicago/London.

Lorenz Bourjot, Martine/Lüger, Heinz Helmut (eds.) (2001): Phraseologie und Phraseodidaktik. Wien.

Lüger, Heinz-Helmut (1997): "Anregungen zur Phraseodidaktik". Beiträge zur Fremdsprachenvermittlung 32: 69-120. 
Petermann, Jürgen (2001): "Razmišljanje o konceptu minimalnih frazeolo_kih slovarjev za tujce". Skripta 5. Zbornik za učitelje slovenščine kot drugegaltujega jezika. Ljubljana: $23-32$.

Šajánková, Monika (2005): "Auswahl der Phraseologismen zur Entwicklung der aktiven phraseologischen Kompetenz". In: Jankovičová, Milada/Mlacek, Jozef/Skladaná, Jana (eds.): Frazeologicke študie IV. Bratislava: 325-340.

Worbs, Erika (1994): Theorie und Praxis der slawisch-deutschen Phraseographie. Mainz.

Wotjak, Barbara (1989): "Ansatz eines modular-integrativen Beschreibungsmodells für verbale Phraseolexeme (PL)". In: Gréciano, Gertrud (ed.): Europhras 88. Phraséologie Contrastive. Actes du Colloque International, Klingenthal-Strasbourg 12-16 mai 1988. Strasbourg: 459-467. 